\title{
Neuronavigation in Endonasal Pituitary and Skull Base Surgery Using an Autoregistration Mask without Head Fixation: An Assessment of Accuracy and Practicality
}

\author{
Nancy McLaughlin ${ }^{1}$ Ricardo L. Carrau ${ }^{2}$ Amin B. Kassam ${ }^{3}$ Daniel F. Kelly ${ }^{1}$ \\ ${ }^{1}$ Brain Tumor Center and Pituitary Disorders Program, John Wayne \\ Cancer Institute at Saint John's Health Center, Santa Monica, \\ California, United States \\ 2 Department of Head and Neck Surgery, Ohio State University, \\ Columbus, Ohio \\ ${ }^{3}$ Department of Neurosurgery, The Ottawa Hospital, Ontario, Canada

\begin{abstract}
Address for correspondence and reprint requests Daniel F. Kelly, M.D., Brain Tumor Center and Pituitary Disorders Program, John Wayne Cancer Institute at Saint John's Health Center, 2200 Santa Monica Blvd., Santa Monica, CA 90404,

United States (e-mail: kellyd@jwci.org).
\end{abstract}

J Neurol Surg A 2012;73:351-357.

\begin{abstract}
Keywords

- endonasal transsphenoidal surgery

- navigation

- endoscopic surgery

- patient positioning

- pituitary adenoma

- skull base tumor

Background Intraoperative navigation is an important tool used during endonasal surgery, which typically requires rigid head fixation. Herein we describe a navigational technique using an autoregistration mask without head fixation.

Material and Methods Prospective evaluation of a surface autoregistration mask used without rigid head fixation in 12 consecutive endonasal endoscopic skull base procedures was performed with patients positioned in a horseshoe head holder. We assessed the accuracy by recording the surface registration error (SRE) and target registration error (TRE). We also noted the time required for installation and the occurrence of system failure. The system's accuracy was validated using a deep target simultaneously viewed with endoscopic.

Results In 12 consecutive endonasal cases performed by a neurosurgeon and ENT team, pathologies included pituitary macroadenomas (9), chordoma (1), craniopharyngioma (1), and sinonasal melanoma (1). Median time required for the registration and accuracy verification was 84 seconds (interval 64 to 129 seconds). The mask stayed on the patient throughout the procedure. The mean SRE was $0.8 \mathrm{~mm}$ (interval 0.6 to $0.9 \mathrm{~mm}$ ). The mean TRE was $0.9 \pm 0.7 \mathrm{~mm}$ and $1.0 \pm 0.8 \mathrm{~mm}$ measured respectively at the beginning and end of the case. In every case, the system was judged accurate by the surgical team using the sphenoid keel or an intrasphenoidal bony septation as a deep target for internal validation. No system failure occurred during these 12 cases.

Conclusion A facial surface autoregistration mask maintained in place throughout surgery without rigid head fixation allows excellent operational accuracy in endonasal pituitary and skull base surgery. This navigation system is practical, reliable, and noninvasive.
\end{abstract}

\section{Introduction}

Intraoperative navigation for intracranial surgery has become increasingly routine and popular within the last two decades.
This technology has significantly contributed to the progression and safety of endonasal pituitary and skull base surgery and clearly serves as a valuable tool in these procedures. In received

June 6, 2011

accepted after revision

May 28, 2012

published online

October 3, 2012
Copyright $\odot 2012$ by Thieme Medical Publishers, Inc., 333 Seventh Avenue, New York, NY 10001, USA. Tel: +1(212) 584-4662.
DOI http://dx.doi.org/ $10.1055 / \mathrm{s}-0032-1326943$. ISSN 2193-6315. 
2002, the American Academy of Otolaryngology/Head and Neck Surgery published recommendations for intraoperative use of image-guided surgery (IGS) for specific pathologies involving the frontal, posterior ethmoidal, and sphenoid sinuses; disease abutting the skull base, orbit, optic nerves, and ICA; CSF leak; and benign and malignant sinonasal neoplasm. ${ }^{1,2}$ Beyond confirmation of midline position, navigation is especially useful for correlation of specific anatomical landmarks in patient space in the axial, coronal, and sagittal planes.

Most frameless navigation systems require rigid head fixation to facilitate accurate registration with scalp fiducials (SFs) or bone fiducials (BFs) or laser surface matching. ${ }^{2-5}$ However, in endonasal pituitary surgery, many surgeons prefer to keep the head only semi-immobilized in a horseshoe head holder that allows slight head movements to facilitate surgeon access and obviates potential postoperative pain and bleeding at pin sites. Until recently, there did not appear to be an IGS system that allowed accurate navigation without rigid head fixation. Since 2005 we have used a noninvasive surface autoregistration mask that adheres to the patient's face and forehead without the need for scalp marking or shaving (Stryker Navigation, Freiburg, Germany). The mask holds 31 lightemitting diodes (LEDs) distributed on one central (nasal) and two lateral arms. In our initial use of the mask over 4 years, patients' heads were fixed in a Mayfield head holder, the data was transferred to a tracker, and the mask was removed after registration. More recently, we have returned to only semirigid immobilization in a horseshoe head holder for many endonasal cases and have fine-tuned use of the autoregistration mask with the communication box attached to the mask. We have now used this technique of facial autoregistration mask with only semirigid immobilization in 50 endonasal cases, and it has become our preferred method. This study assessed the accuracy and the practicality of this navigation technique in endonasal endoscopic surgery without rigid head fixation.

\section{Methods}

\section{Patient Cohort}

All consecutive patients undergoing surgery at Saint John's Health Center for sellar and/or parasellar pathologies from October to December 2010 were included in this study. The surgical team comprised a neurosurgeon and an ear, nose, and throat (ENT) surgeon. The endonasal route was used in all cases using either an endoscope-assisted or a purely endoscopic technique. Patient consent for the use of the navigation system was obtained at the time of surgical consent. The Institutional Review Boards of each institution approved this retrospective study of patient data.

\section{Technique Description}

Once the patient is under general anesthesia, the head is placed upon a padded horseshoe head holder with 15 degrees of rightward rotation and a slight leftward tilt to facilitate surgeon access from the patient's right side. Greater degrees of head extension are used for lesions extending anterior to the sella in the frontal fossa.
All patients undergo a pre-operative sellar magnetic resonance imaging (MRI) and an additional T1 post-gadolinium thin-slice (2-mm cuts) navigational sequence. The navigational MRI is uploaded into the Stryker Navigation System (Stryker Instruments, Kalamazoo, Michigan), with axial, coronal, and sagittal views, and the optical detection camera is appropriately positioned, typically three to five feet above and behind the patient's head. The optical camera has a bird's- eye view of the registration mask and communication box, ensuring constant tracking. Both surgeons stand to the right of the patient's head and torso. Only occasionally does the person driving the endoscope need to slightly change the angle with which the scope is being held to avoid line- ofsight obstruction to the mask. This problem has not been found to be a limitation but is a well-appreciated issue common to all optical tracking navigation systems.

As shown in - Fig. 1, the surface auto-registration mask is positioned over the forehead, nose, and upper face (Stryker Navigation, Freiburg, Germany). The communication box is attached to the mask and then supported with $4 \times 4$ pads and taped to the horseshoe. This securing of the box prevents it from pulling and distorting the mask on the forehead, which will otherwise distort the registration process with loss of accuracy. It also prevents movement of the communication box during the case, should the patient be rotated laterally.

The communication box is then activated. The optical infrared camera must recognize at least 21 of the 31 LEDs of the mask. For optimal results, all except one LED should be seen on each arch. Before proceeding with registration, care must be taken to make sure that the endotracheal tube tape is not displacing or distorting the cheeks or nose skin to which the mask is attached. Also, there should be no tape covering the eyes. Once the system has established a correlation between the image data and patient data, the accuracy is initially qualitatively assessed by touching five anatomical landmarks. When assessing the correspondence between image space and patient space, care must be taken to gently apply the tip on the patient's skin without any pressure because this can lead to erroneous interpretation of the accuracy. The anatomical landmarks used in this study include the left outer canthus (point 1), left inner canthus (point 2), the nasion (point 3), the right inner canthus (point 4), and the right outer canthus (point 5) (-Figs. 2, 3). The distance between the actual and virtual target is assessed on the axial, coronal, and sagittal planes. If the distance was consistently less than $1 \mathrm{~mm}$ through the verification process, the registration was accepted. The system provides a surface registration error (SRE) in millimeters. The target registration error (TRE) (or operational accuracy) was assessed at the beginning and end of the case by measuring the distance between the tip of the navigation wand and the skin as measured in millimeters on the axial, coronal, and sagittal views for each landmark. The Stryker Navigation System allows measuring submillimetric distances on the processed axial, coronal, and sagittal sequences.

The autoregistration mask is left in place throughout the case. The eyes can then be protected, taking care not to cover the top arches with tape. After registration, only the LEDs on the top arches of both lateral arms emit a signal for 


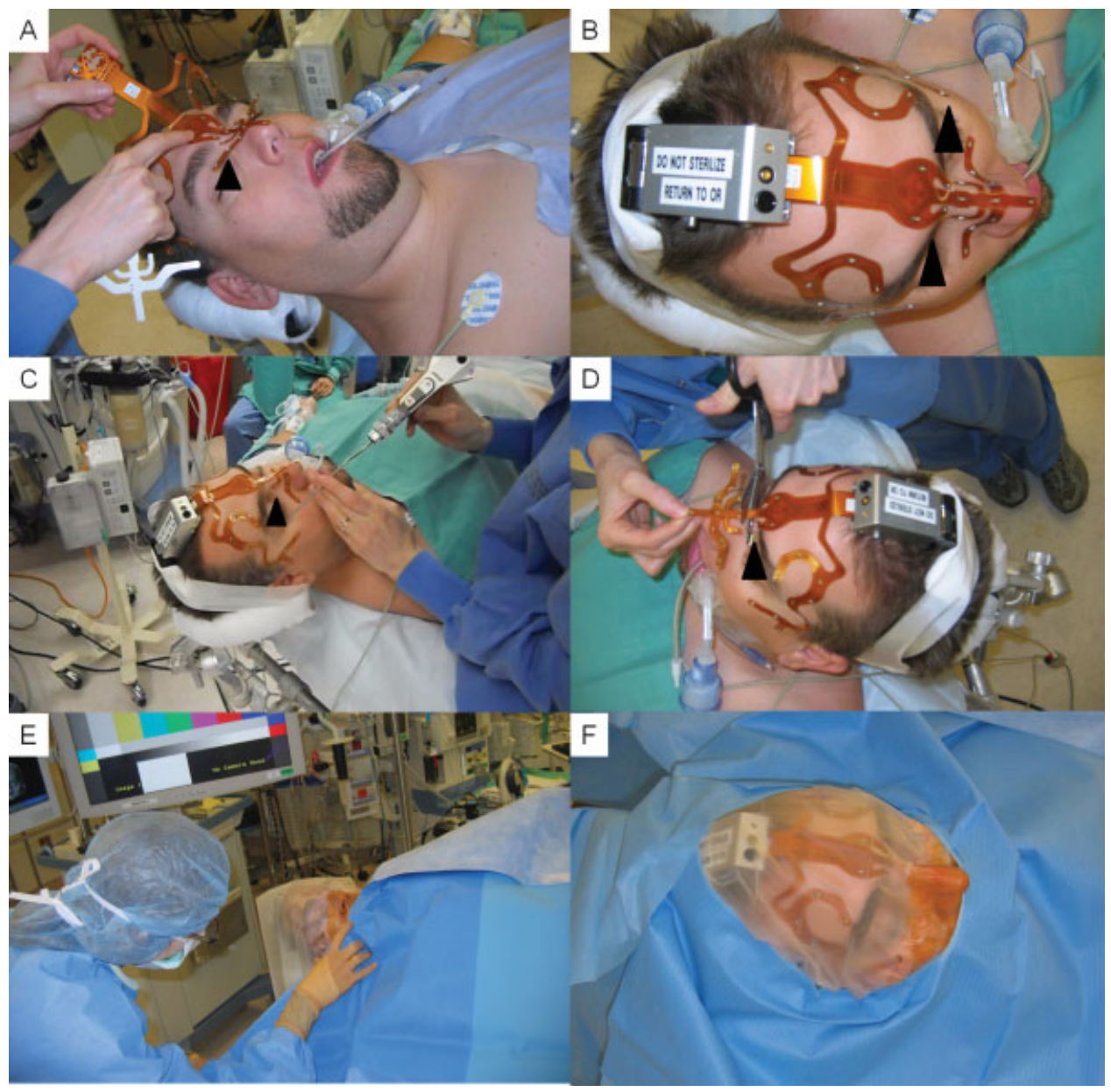

Figure 1 Overview of the use of the autoregistration mask without head fixation.

continuous navigation. Any pulling or pushing on the mask transmitted to the superior arches can contribute to loss of operational accuracy. Therefore, the branch of LEDs that extends along the nose is cut after registration is completed. This eliminates potential navigational error from nasal distortion by the endoscope, endonasal speculum, or other instruments that can distort the nasal skin contour.

Since the mask and the communication box must remain visible to the optical camera throughout the case, a clear sterile drape is required. The only opaque drape that covers the facial skin is below the nares to cover the mouth. The rest of the drapes are stuck to the clear drape, preventing any distortion by the weight of the drapes. The rest of the draping proceeds as usual.

At the beginning of each case, the system's accuracy is also validated using the midline sphenoid keel or an intrasphenoidal bony septation visualized directly with the endoscope. The system is evaluated as accurate by the surgical team if the operational accuracy was $<1 \mathrm{~mm}$. Intraoperative navigation is used regularly throughout endonasal procedures. Practicality was also evaluated by measuring the time taken from activation of the communication box to acceptance of registration. This represents the time in the operating room (OR) uniquely dedicated to the use of the navigation system. Failure of the system is also a measure of practicality. Intraoperative complications were also noted.

\section{Results}

Over a 7-week period from October to December 2010, 12 consecutive patients underwent surgery for a sphenoid, sellar, parasellar pathology including pituitary macroadenomas $(n=9)$, chordoma $(n=1)$, craniopharyngioma $(n=1)$, and sinonasal melanoma $(n=1)$. Four of these surgeries were for recurrent pathologies ( - Table $\mathbf{1}$ ).

Using the five anatomical landmarks detailed in the methods, the median time required for registration and accuracy verification took 84 seconds varying between 64 to 129 seconds ( $\boldsymbol{- T a b l e} \mathbf{1}$ ). No system failure occurred in these 12 cases and no surgical complications occurred.

The mean SRE recorded by the neuronavigational software was $0.8 \mathrm{~mm}$ (interval 0.6 to $0.9 \mathrm{~mm}$ ). At the beginning of the case, the mean TRE was $0.9 \pm 0.7 \mathrm{~mm}$ and the median was $1 \mathrm{~mm}$ (interval of 0.2 to $3 \mathrm{~mm}$ ). The mean TRE at the end of the procedure was $1.0 \pm 0.8 \mathrm{~mm}$ and the median was $0.5 \mathrm{~mm}$ (interval of 0.2 to $3 \mathrm{~mm}$ ). In $44 \%$ of readings, there was no change of the TRE between the initial values and those measured at the end of the procedure. The average absolute difference between initial and final TRE was $0.7 \pm 0.7 \mathrm{~mm}$ and the median was $0.8 \mathrm{~mm}$ (interval of 0 to $1.8 \mathrm{~mm}$ ). For all the cases in this study population, both surgeons of the surgical team were satisfied with the intraoperative navigation operational accuracy verified at the beginning of surgery 
354 Navigation with the Autoregistration Mask McLaughlin et al.

Table 1 Summary of Data

\begin{tabular}{|l|l|l|l|l|l|}
\hline Case & Pathology & Time $(\mathrm{sec})$ & SRE $^{\mathrm{a}}(\mathrm{mm})$ & TRE $(\mathrm{mm})^{\mathrm{b}}$ pre-op & ${\text { TRE }(\mathrm{mm})^{\mathrm{b}} \text { post-op }}^{\text {Th }}$ \\
\hline 1 & Recurrent chordoma & 92 & 0.6 & 0.6 & 1.3 \\
\hline 2 & Pituitary macroadenoma & 92 & 0.9 & 1.5 & 2.08 \\
\hline 3 & Recurrent pituitary macroadenoma & 129 & 0.8 & 0.83 & 1.75 \\
\hline 4 & Recurrent sinonasal melanoma & 71 & 0.8 & 0.2 & 0.4 \\
\hline 5 & Pituitary macroadenoma with apoplexy & 58 & 0.6 & 1.1 & 1.07 \\
\hline 6 & Pituitary macroadenoma & 59 & 0.8 & 0.92 & 1.04 \\
\hline 7 & Craniopharyngioma & 88 & 0.8 & 0.47 & 0.52 \\
\hline 8 & Pituitary macroadenoma & 78 & 0.6 & 0.6 & $\mathrm{~N} / \mathrm{A}^{\mathrm{c}}$ \\
\hline 9 & Pituitary macroadenoma & 77 & 0.8 & 0.84 & 0.90 \\
\hline 10 & Pituitary macroadenoma & 64 & 0.8 & 0.52 & 1.08 \\
\hline 11 & Pituitary macroadenoma & 90 & 0.8 & 0.8 & 0.52 \\
\hline 12 & Pituitary macroadenoma & 133 & 0.9 & 0.88 & 0.56 \\
\hline
\end{tabular}

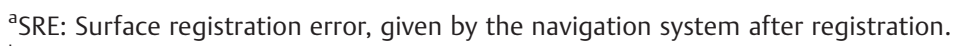

b Target registration error (TRE): Measures the distance between the tip of the navigation wand and the target. The mean value for each patient is presented.

'The postoperative TRE values were not available (N/A) for this patient; however, excellent correlation was noted between the image point and the patient point throughout the case. The calculations presented in the result take this into consideration.

using a deep bone landmark (TRE $<1 \mathrm{~mm}$ ). However, in two cases the navigation system appeared to be intermittently inaccurate. Intraoperative troubleshooting found that this occurred when some of the LEDs from the mask were hidden either by the surgeon's hand, the endoscope cables, or the drapes. Once this was addressed and all the LEDs could be seen by the optical camera, navigation was once again accurate.

\section{Discussion}

\section{Navigation and Endonasal Surgery}

Throughout the years, numerous navigation systems have been used in image-guided surgeries. Traditional intraoperative fluoroscopy provides sagittal plane bony orientation and navigation in transsphenoidal surgery. Although it allows one to work without head fixation, its monoplanar bony information is clearly limited. Current computed tomography (CT) or MRI-based navigation systems provide a much greater degree of anatomical detail without radiation. In the field of sinonasal and endonasal endoscopic skull base surgery, these systems appear to have increased the safety of surgery and facilitated the learning curve for many surgeons. ${ }^{6}$ In transcranial surgery, these systems have allowed tailoring of approaches by planning smaller yet strategically positioned craniotomies, helping advance the concept of keyhole surgery. Although navigation does not replace a thorough knowledge of the sinonasal and endonasal skull base anatomy, provided the system has intraoperative accuracy, it allows instantaneous correlation of patient space and image space in three planes. It also enables surgeons to readjust their three-dimensional (3D) perception, which is especially useful in cases with anatomical variations or prior surgery and thereby helps prevent catastrophic complications. ${ }^{6}$
The BFs represent the gold standard of pair-point registration, yielding the highest navigational accuracy. ${ }^{2,3}$ However, this technique is also the most invasive, potentially causing discomfort to the patient as well as an additional CT scan and its associated radiation. ${ }^{2,5}$ Some less invasive registration methods and navigation systems may result in satisfactory levels of accuracy (in the 1.0- to $2.0-\mathrm{mm}$ range) for specific regions of interest such as the midface and skull base. ${ }^{2,7} \mathrm{We}$ have also tried using a headband technique (BrainLab, Feldkirchen, Germany) without head fixation, which did not prove sufficiently reliable or accurate.

\section{Using the Autoregistration Mask without Head Fixation}

The autoregistration mask is a simple, noninvasive surface registration technique. It requires no special preparation at the patient's bedside because it is installed after the patient is intubated and requires no additional imaging after the mask is positioned. More recently, as shown in this study population, we have stopped fixing the head in pins and proceed with free head navigation, leaving the mask in place throughout the entire procedure. In a technical note on endonasal combined microscopic endoscopic surgery for removal of pituitary adenomas, Al-Mefty and colleagues mention using the mask for registration without fixation of the patient's head. ${ }^{8}$ They state that the mask is an excellent adjunct for navigating during pituitary surgery because of its easy and intuitive use. More recently, Makieses compared in a cadaver model the operational accuracy of the autoregistration mask with that obtained with SFs or BFs. ${ }^{5}$ The accuracy for external anterior targets was $1.96,3.12$, and $3.20 \mathrm{~mm}$ for BF, SF, and autoregistration mask, respectively; however, when reaching internal targets, the autoregistration mask was associated with an operational accuracy $(2.41 \mathrm{~mm})$ statistically superior 


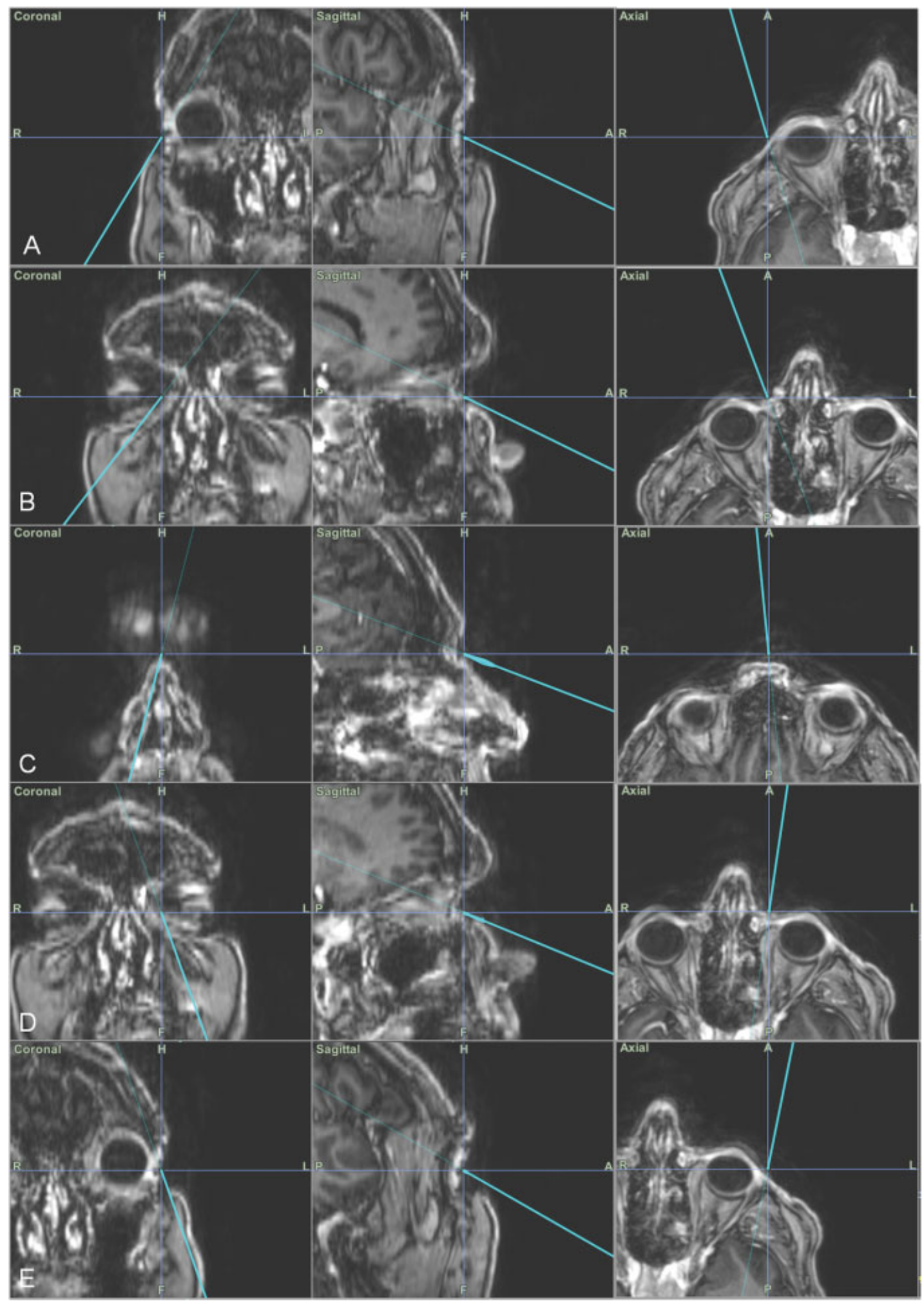

Figure 2 Example of navigation accuracy at the beginning of the procedure (case 12). Views in coronal, sagittal and axial planes at the right outer canthus (A); right medial canthus (B); nasion (C); left medial canthus (D); left outer canthus (E).

to that associated with $\mathrm{BF}(2.91 \mathrm{~mm})$ or $\mathrm{SF}(3.83 \mathrm{~mm}) .^{5}$ This is the first anatomical study that assessed the accuracy of the autoregistration mask. ${ }^{5}$ The authors mention that, given its incorporated tracking system that can be seen by the optical camera, rigid fixation of the patient's head may be obviated. Additionally, they state that rigid fixation may cause displace- ment or distortion of the skin, alter the integration process of image-patient coordinates, and result in decreased accura$\mathrm{cy}^{4,9}$ To our knowledge, this study is the first clinical report showing feasibility and accuracy of using the autoregistration mask throughout endonasal surgery without rigid head fixation. 


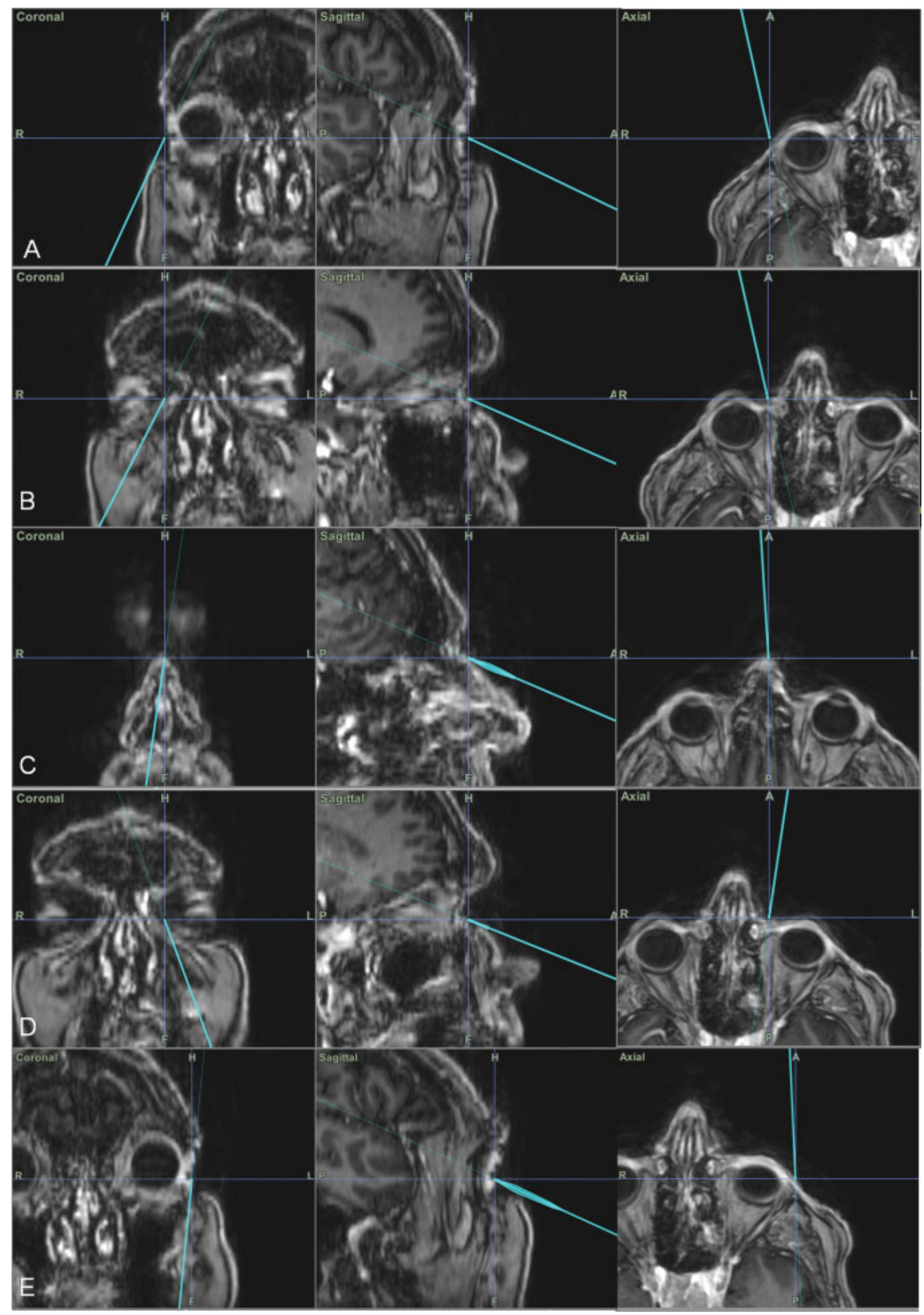

Figure 3 Example of navigation accuracy at the end of the procedure (case 12). Views in coronal, sagittal and axial planes at the right outer canthus (A); right medial canthus (B); nasion (C); left medial canthus (D); left outer canthus (E).

\section{Accuracy and Practicality}

In the clinical setting, navigation systems reach operational accuracies between 0.5 and $2.77 \mathrm{~mm}, 2,10,11$ and several reports have stated that an operational accuracy (TRE) of 1 to
$2 \mathrm{~mm}$ is required for safe use of a navigation system. ${ }^{2,7}$ For each navigation system, numerous factors can influence the operational accuracy, including preoperative image quality, the registration procedure, and the navigation system. ${ }^{9,12,13}$ 
Intraoperative events can also modify the accuracy, such as movement of the tracking device. Accuracy values also vary depending on if the assessment was performed on cadavers, ${ }^{5}$ skull models, ${ }^{2}$ or patients. ${ }^{14,15}$ This study demonstrates that satisfactory accuracy can be obtained by using the autoregistration mask without head fixation (free-head) while maintaining excellent accuracy: a SRE of $0.8 \mathrm{~mm}$ (interval 0.6 to $0.9 \mathrm{~mm}$ ) and a mean TRE of $0.9 \mathrm{~mm}$ and $1.0 \mathrm{~mm}$ measured respectively at the beginning and end of the case. In almost half of the readings (44\%), there were no shifts between the initial and final TRE. Numerous adjustments were brought since the beginning of the use of the autoregistration mask. The set-up and technique as described in the methods is simple and rapid. The small technical details-including cutting the nasal branch of the LED display, making sure the eye dressing does not cover the superior lateral arches of the mask, using a clear drape, and preventing the LEDs from being hidden from the scope cables-together ensure the best operational accuracy throughout surgery. Neuromonitoring can be used in the presence of the autoregistration mask and the communication box but requires that the scalp sensors be positioned further posterior because of the possible noise in the somatosensory evoked potential (SSEP) signal. Postoperatively, we have observed fewer patients reporting scalp and head pain, although we did not objectively document this in the current study.

\section{Conclusion}

Use of the surface autoregistration mask in endonasal pituitary and skull base tumor surgery without head fixation enables satisfactory operational accuracy throughout the procedure, and is practical, reliable, and noninvasive. Further assessment will help determine in which surgical procedures this system is most beneficial and how to improve this registration technique and navigation system.

\author{
Conflict of Interest \\ None
}

\section{References}

1 Wise SK, DelGaudio JM. Computer-aided surgery of the paranasal sinuses and skull base. Expert Rev Med Devices 2005;2:395-408

2 Grauvogel TD, Soteriou E, Metzger MC, Berlis A, Maier W. Influence of different registration modalities on navigation accuracy in ear, nose, and throat surgery depending on the surgical field. Laryngoscope 2010;120:881-888

3 Eggers G, Mühling J, Marmulla R. Image-to-patient registration techniques in head surgery. Int J Oral Maxillofac Surg 2006;35; 1081-1095

4 Mitsui T, Fujii M, Tsuzaka M, Hayashi Y, Asahina Y, Wakabayashi T. Skin shift and its effect on navigation accuracy in image-guided neurosurgery. Radiological Phys Technol 2011;4:37-42

5 Makiese O, Pillai P, Salma A, Sammet S, Ammirati M. Accuracy validation in a cadaver model of cranial neuronavigation using a surface autoregistration mask. Neurosurgery 2010;67(3, Suppl Operative):ons85-ons90, discussion ons90

6 Casiano RR, Numa WA Jr. Efficacy of computed tomographic image-guided endoscopic sinus surgery in residency training programs. Laryngoscope 2000;110:1277-1282

7 Cartellieri M, Vorbeck F. Endoscopic sinus surgery using intraoperative computed tomography imaging for updating a threedimensional navigation system. Laryngoscope 2000;110(2 Pt 1): 292-296

8 Al-Mefty O, Pravdenkova S, Gragnaniello C. A technical note on endonasal combined microscopic endoscopic with free head navigation technique of removal of pituitary adenomas. Neurosurg Rev 2010;33:243-248, discussion 248-249

9 Watanabe Y, Fujii M, Hayashi Y, et al. Evaluation of errors influencing accuracy in image-guided neurosurgery. Radiological Phys Technol 2009;2:120-125

10 Schlaier J, Warnat J, Brawanski A. Registration accuracy and practicability of laser-directed surface matching. Comput Aided Surg 2002;7:284-290

11 Caversaccio M, Nolte LP, Häusler R. Present state and future perspectives of computer aided surgery in the field of ENT and skull base. Acta Otorhinolaryngol Belg 2002;56:51-59

12 Snyderman C, Zimmer LA, Kassam A. Sources of registration error with image guidance systems during endoscopic anterior cranial base surgery. Otolaryngol Head Neck Surg 2004;131:145-149

13 Steinmeier R, Rachinger J, Kaus M, Ganslandt O, Huk W, Fahlbusch R. Factors influencing the application accuracy of neuronavigation systems. Stereotact Funct Neurosurg 2000;75:188-202

14 Greenfield JP, Howard BM, Huang C, Boockvar JA. Endoscopic endonasal transsphenoidal surgery using a skull reference array and laser surface scanning. Minim Invasive Neurosurg 2008;51:244-246

15 Eliashar R, Sichel JY, Gross M, et al. Image guided navigation system-a new technology for complex endoscopic endonasal surgery. Postgrad Med J 2003;79:686-690 